\title{
Uso e diversidade de plantas medicinais em uma comunidade quilombola no Raso da Catarina, Bahia
}

\author{
Thiago Bezerra Gomes ${ }^{1}$ e Fábio Pedro Souza de Ferreira Bandeira ${ }^{2,3}$
}

Recebido em 26/03/2012. Aceito em 25/06/2012

\begin{abstract}
RESUMO
(Uso e diversidade de plantas medicinais em uma comunidade quilombola no Raso da Catarina, Bahia). A contribuição dos povos afrodescendentes é de extrema importância para a formação da cultura brasileira. Esses povos são em parte representados pelas comunidades remanescentes de quilombos, que mantém costumes e conhecimentos sobre utilização e manejo dos recursos vegetais. Esta pesquisa teve como objetivos inventariar as plantas medicinais utilizadas bem como medir a saliência cultural das mesmas, na comunidade Casinhas, município de Jeremoabo, estado da Bahia, localizada numa região de Caatinga. Para coleta dos dados foram realizadas entrevistas semi-estruturadas com sete pessoas reconhecidas pela comunidade como os maiores detentores do conhecimento sobre plantas. Os resultados indicam que 87 espécies são utilizadas na medicina tradicional local, merecendo destaque Poincianella pyramidalis (catingueira), que apresentou maior freqüência de citação e maior valor de saliência. O sistema digestório teve o maior número de indicações de plantas relacionadas às suas afecções (21 espécies); as folhas (36\%) e as cascas (30\%) foram as partes mais citadas nas indicações terapêuticas; o chá foi a forma de uso mais indicada (49\%). A comunidade estudada depende diretamente dos recursos vegetais para suas práticas de cura. Os resultados dessa pesquisa podem servir como base para bioprospecção bem como subsídio para seleção de espécies da caatinga prioritárias para estudos posteriores de ecologia de populações, visando o seu uso e manejo sustentável.
\end{abstract}

Palavras-chave: Caatinga, comunidades negras, índice de saliência

\begin{abstract}
(The use and diversity of medicinal plants in a quilombola community in Raso da Catarina, Bahia). Contributions from Afro-descendant peoples have been hugely important in the formation of Brazilian culture. These peoples are partly represented by remnant quilombo communities, which maintain their habits and knowledge about the use and management of plant resources. This research aimed to inventory the medicinal plants used, and to measure the cultural salience of these plants in the Casinhas community of the Jeremoabo municipality, in the state of Bahia (which is situated in a caatinga region). For data collection, we conducted semi-structured interviews with seven people recognized by the community as having the most knowledge about plants. The results indicate that 87 species are used in traditional local medicine, with an emphasis on Poincianella pyramidalis (catingueira), which had the greatest citation frequency and the greatest salience. Diseases of the digestive system had the most plant indications (21 species), leaves (36\%) and bark (30\%) were the plant parts most often cited for therapeutic use, and tea was the most frequently indicated mode of use (49\%). The studied community depends directly on plant resources for their curing practices. The results of this research may serve as a basis for bioprospecting and may also be of benefit in the selection of priority caatinga species for future studies about the ecology of populations, aimed at their sustainable use and management.
\end{abstract}

Key words: Afrobrasilian community, Caatinga, salience index

\footnotetext{
${ }^{1}$ Universidade do Estado da Bahia, Departamento de Educação, Programa de Pós-Graduação em Ecologia Humana e Gestão Socioambiental, Paulo Afonso, BA, Brasil

${ }^{2}$ Universidade Estadual de Feira de Santana, Departamento de Ciências Biológicas, Núcleo de Pesquisa em Ambiente Sociedade e Sustentabilidade, Feira de Santana, BA, Brasil

${ }^{3}$ Autor para Correspondência: fpbandeira@gmail.com
} 


\section{Introdução}

A Etnobotânica é definida como o estudo das interrelações, ecológicas, evolucionárias e simbólicas das sociedades humanas, passadas e presentes, com as plantas (Alexiades 1996; Albuquerque 2005). Os estudos realizados nessa área são de alta relevância científica, econômica e social e têm mostrado sua importância para a conservação e manejo da vegetação nas regiões tropicais, especialmente no Brasil, uma vez que seu território abriga uma das floras mais ricas do planeta, da qual $99 \%$ são desconhecidas quimicamente (Gottlieb et al. 1998; Simões at al. 1998) aliado ao fato de que o país apresenta uma alta diversidade cultural (Toledo 2001).

Dentre os diversos povos e comunidades tradicionais presentes no território nacional, a região nordeste, sobretudo a Bahia, apresenta o maior número de comunidades quilombolas em todo o Brasil (Anjos, 2000), porém há poucos estudos desenvolvidos sobre essas comunidades, inclusive trabalhos de etnobotânica. (Albuquerque 2001; Franco \& Barros 2006; Monteles \& Pinheiro 2007; Vieira et al 2008; Guimarães 2009; Almeida \& Bandeira 2010).

Muitos foram os povos africanos importados no sistema escravista do século XVI, durante o período de colonização das Américas. Esse sistema teve particularidades substanciais no Brasil em relação ao resto do continente americano, uma vez que a sociedade escravista conseguiu se estabilizar e se desenvolver nessa região, verificando-se a continuidade de importação de escravos por muitos séculos. Conforme assinalado por Schmitt et al. (2002), os grupos hoje considerados remanescentes de comunidades de quilombo se constituíram a partir de uma grande diversidade de processos, desde movimentos insurrecionais, heranças, doações, recebimento e compra efetiva de terras ou pela simples permanência nas terras que ocupavam e cultivavam no interior dos latifúndios, tanto durante a vigência do sistema escravocrata quanto após sua derrocada.

Vale ressaltar que os povos africanos não foram responsáveis somente pelo povoamento do território brasileiro e pela mão-de-obra escrava, eles têm marcado decisivamente a nossa formação social e cultural, e as diversas configurações socioculturais afrobrasileiras têm sido preservadas e recriadas, a despeito das políticas contrárias a sua permanência (Anjos, 2000. Ainda assim, a sociedade nacional tem se referido aos quilombos sempre no passado, como se estes não fizessem mais parte da vida do país. Os registros do conhecimento tradicional das comunidades negras no Brasil ainda são escassos, porém, muitas dessas comunidades mantêm ainda tradições que seus antepassados trouxeram da África, como práticas agrícolas, de cuidado da saúde física e espiritual, práticas religiosas bem como técnicas de mineração, arquitetura e construção, além do artesanato, da culinária, de relações comunitárias de uso da terra, dentre outras formas de expressão cultural (Anjos, 2000). Enfatiza-se a importância, nesse contexto, da realização de estudos desses saberes e da relação dessas comunidades com o meio ambiente.
Falando especificamente sobre o conhecimento tradicional sobre as plantas medicinais, vale ressaltar que esses conhecimentos vêm sofrendo ameaças constantes para sua manutenção, devido à influência direta da medicina moderna e pelo desinteresse dos jovens das comunidades, interrompendo assim o processo de transmissão de saberes entre as gerações (Amorozo 1996). Desse modo, o presente estudo teve como principal objetivo inventariar as plantas utilizadas na medicina tradicional de uma comunidade quilombola, no Raso da Catarina, pertencente ao Bioma Caatinga, e assim, estabelecer bases para a proteção e valorização desse conhecimento.

\section{Material e métodos}

\section{Área de estudo}

O município de Jeremoabo está situado no Nordeste do estado da Bahia, distando $370 \mathrm{~km}$ da capital do Estado. Seu nome tem origem tupi e significa "plantação de abóbora" (http://www.jeremoabo.com.br/informacoes/historia). Possui uma área de $4.788,4 \mathrm{~km}^{2}$, com altitude de $275.7 \mathrm{~m}$; clima semi-árido com chuvas de maio a julho e alto risco de seca, sendo a temperatura média anual de $24^{\circ} \mathrm{C}$. A economia está baseada na agricultura, pecuária e avicultura. A população do município tem cerca de 40.000 habitantes (IBGE, 2008). Tornou-se Vila pelo decreto de 25 de outubro de 1831, passando a condição de cidade no ano de 1925, pelo decreto $n^{\circ} .1775$ de 06 de julho de 1925 .

A área é coberta por uma vegetação do tipo caatinga que não se apresenta homogênea, mas como um mosaico descontínuo, com predominância de vegetação de aspecto arbóreo aberto, às vezes mais denso ou mais rarefeito e de menor porte (PDRS 2000), apresenta também áreas de contato entre cerrado-caatinga-floresta estacional, contato cerrado-caatinga, caatinga arbórea sem palmeiras, caatinga arbórea densa sem palmeiras, caatinga arbórea aberta com palmeiras (CEI 1994). Parte importante do município de Jeremoabo está inserida na Estação Ecológica do Raso da Catarina (ESEC). A Estação Ecológica Raso da Catarina situa-se à margem esquerda do rio Vaza Barris e à margem direita do Rio São Francisco, abrangendo uma área de 105.282,00 ha (IBAMA, 2005), e estende- se sob as coordenadas $09^{\circ} 33^{\prime}-09^{\circ} 54^{\prime} \mathrm{S}, 38^{\circ} 29^{\prime}-38^{\circ} 44^{\prime} \mathrm{W}$. A ESEC encontra-se assentada sobre uma bacia sedimentar no domínio das Caatingas, exibindo fisionomia arbórea, arbustiva e arbustivo-arbórea, raramente ocorrendo matas isoladas (IBAMA, 2005). De acordo com o Ministério do Meio Ambiente-MMA (2002) a ESEC Raso da Catarina é uma área altamente prioritária para conservação.

Caracterização da comunidade - A comunidade quilombola de Casinhas está localizada na BR 110, a 36 km de distância da sede e tem aproximadamente 80 famílias ( 300 pessoas). De acordo com a história oral do lugar, os moradores que hoje vivem em Casinhas são remanescentes daqueles que vieram 
fugidos do trabalho escravo e dos grandes coronéis. Ao chegarem, eles construíram algumas "casinhas" para se abrigarem, daí originou-se o nome da comunidade. Durante a pesquisa, a comunidade passava por um processo de reconhecimento como quilombola, porém Casinhas já havia sido identificada por Anjos (2000) durante a realização da primeira configuração espacial dos remanescentes de antigos quilombos no Brasil. No dia 06/07/2010, a comunidade foi oficialmente reconhecida pela Fundação Palmares (Código do IBGE - 2018100). A comunidade possui uma escola de ensino fundamental - $1^{\circ}$ Ciclo. Também possui associação comunitária. A festa mais importante que realizam é a do Padroeiro São Jerônimo. Casinhas é a comunidade do município de Jeremoabo que dispõe da maior quantidade de fontes de água: além das quatro nascentes das quais ainda brotam água, há também um riacho, "barreiros" (depressões no solo onde se acumulam água da chuva), açude e alguns poços de água doce. $\mathrm{O}$ atendimento médico é precário e quando os moradores necessitam de assistência recorrem aos postos de saúde da rede pública existentes na sede municipal e em outros municípios vizinhos. Diante desse cenário, o uso de plantas medicinais pela comunidade para tratar suas doenças se constitui em uma das poucas alternativas de promoção da saúde.

\section{Coleta e análise de dados}

As primeiras visitas tiveram como principal objetivo conhecer a comunidade, estabelecer o contato inicial com os moradores e identificar seus hábitos e modo de vida. Juntamente com a associação de moradores e lideranças comunitárias se caracterizou a área de estudo. Durante esse período de reconhecimento foram estabelecidos contatos prévios com as famílias para esclarecimento dos objetivos da pesquisa. Foram realizadas sete visitas, com duração de quatro dias cada, no período entre janeiro de 2009 e julho de 2010.

A coleta dos dados foi feita através de entrevistas semi-estruturadas, nas quais se interrogou sobre as plantas reconhecidas para o tratamento de enfermidades e manutenção da saúde, formas de uso e partes da planta utilizadas bem como a indicação terapêutica e local de obtenção. As indicações foram agrupadas com base na classificação das doenças proposta pela Organização Mundial de Saúde (OMS 2000); as doenças ou estados que não puderam ser incluídos nesta classificação geral foram agrupados na categoria "doenças culturais" ou "outros".

Para a seleção dos entrevistados que participaram desta pesquisa, utilizou-se a técnica "bola de neve" (ou "Snow ball") (Bailey 1994), buscando aqueles moradores que fossem os mais antigos da comunidade e que possuíssem amplo conhecimento acerca da utilização de plantas medicinais. Foram identificados sete moradores, reconhecidos pela comunidade como os conhecedores locais detentores do maior acervo de conhecimento etnobotânico, colaboradores dessa pesquisa, sendo quatro homens e três mulheres.

Durante a realização das entrevistas se pediu aos entrevistados que listassem as plantas de acordo com a sua importância. A lista livre obtida permitiu buscar informações específicas sobre um domínio cultural da comunidade estudada (Bernard 1988; Weller \& Romney 1988). Esta lista inclui desse modo, as espécies culturalmente relevantes, pois faz inferências sobre a estrutura cognitiva do informante a partir da ordem em que o mesmo recorda-se e coloca os elementos em dada ordem. A partir da ordenação das espécies feita por cada informante, os dados foram convertidos em um ranking invertido (onde o último item de cada lista recebeu o valor igual a 1 , o penúltimo igual a 2 e assim sucessivamente até o primeiro item de cada lista) e divididos pelo número total de itens em cada lista livre, atribuindo-se um Valor de Saliência (S) para cada item. Para tabulação dos dados utilizou-se o programa ANTROPAC 4.0 (Borgatti 1992), que calcula o somatório de saliência das espécies em comum de todas as listas livres $\left(\sum\right.$ de $\left.S\right)$ e o valor médio de saliência da totalidade dos dados $\left(\sum\right.$ de $S / n$, onde $\mathrm{n}=7$ ). Tal método permite analisar quais espécies são mais salientes, ou seja, as mais importantes e reconhecidas pelos informantes-chaves, além de identificar a variação no conhecimento sobre o domínio estudado (Quinlan 2005).

Após a realização das entrevistas foram realizadas visitas trimestrais à comunidade para coleta de material botânico com o auxílio dos colaboradores locais para o reconhecimento das espécies in loco. As amostras foram coletadas e processadas de acordo com a metodologia proposta por Mori et al. (1989). O material foi depositado no Herbário da Universidade Estadual de Feira de Santana (HUEFS) e identificado com ajuda de especialistas ou por meio de comparação com exsicatas. O sistema adotado para a classificação das famílias botânicas foi o APG III (2009).

\section{Resultados e discussão}

Os sete entrevistados citaram 90 nomes vernaculares de plantas, que são potencialmente utilizadas como medicinais na comunidade de Casinhas, que correspondem a 87 espécies. As famílias que apresentaram maior número de espécies foram Fabaceae (11 espécies), Asteraceae (seis espécies), Anacardiaceae (cinco espécies), seguidas por Euphorbiaceae, Lamiaceae, Caesalpinaceae, Mimosaceae e Poaceae, estas últimas, todas com quatro espécies. Resultados semelhantes foram encontrados em outros estudos realizados na região Nordeste do Brasil (Almeida \& Albuquerque 2002; Teixeira \& Melo 2006; Albuquerque \& Andrade 2002; Albuquerque 2009). Os dados referentes aos recursos vegetais utilizados para fins medicinais estão sistematizados na Tabela 1.

Entre as espécies identificadas como medicinais pela comunidade destacam-se as árvores como o hábito de crescimento mais representativo (39 espécies), mas o número de espécies herbáceas também se mostra bastante expressivo (20 espécies). Aparecem na seqüência os arbustos (13), subarbustos (7) e trepadeiras (4), indicando a importância dos diversos estratos da vegetação nas práticas terapêuticas locais. 


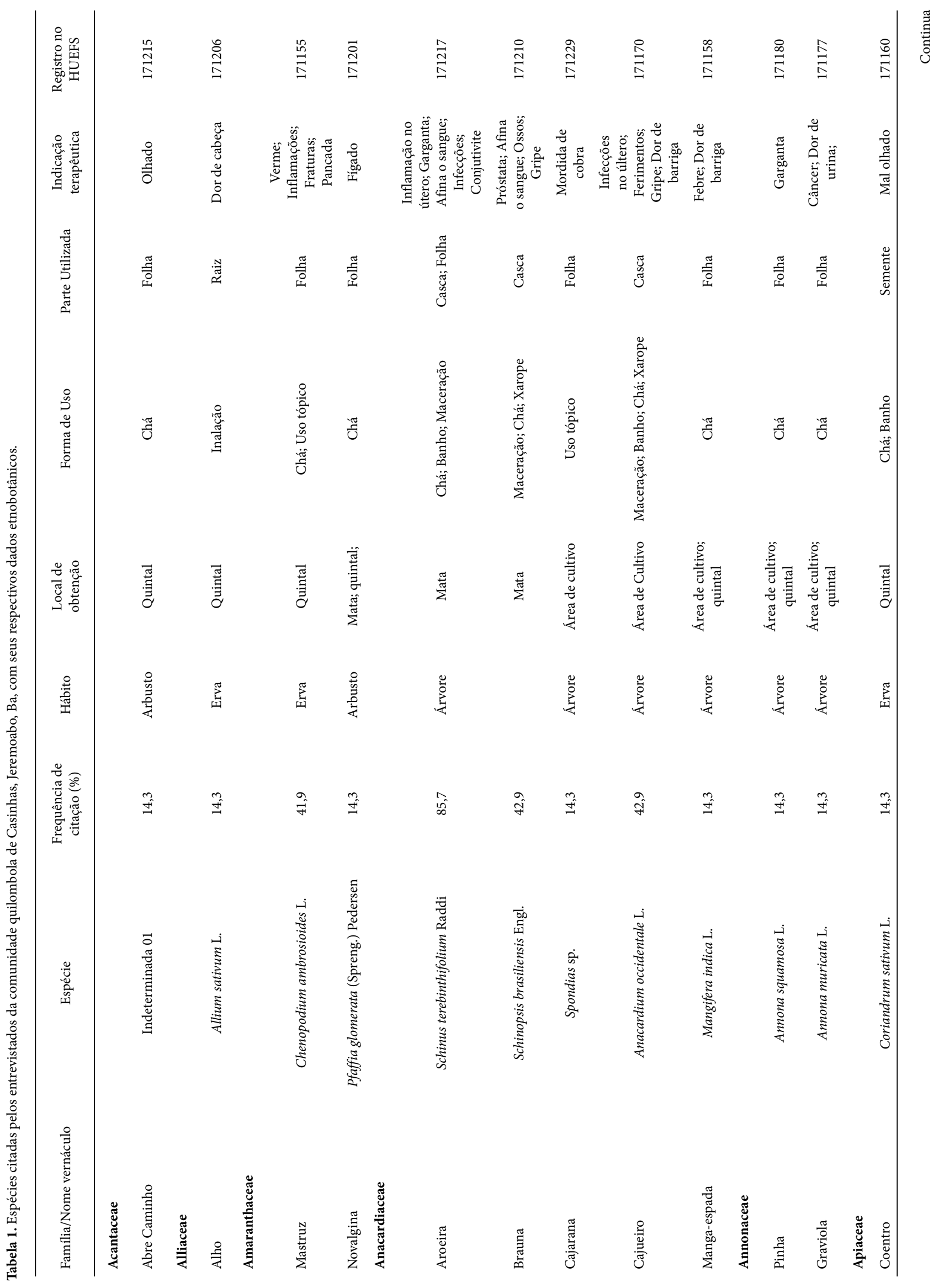




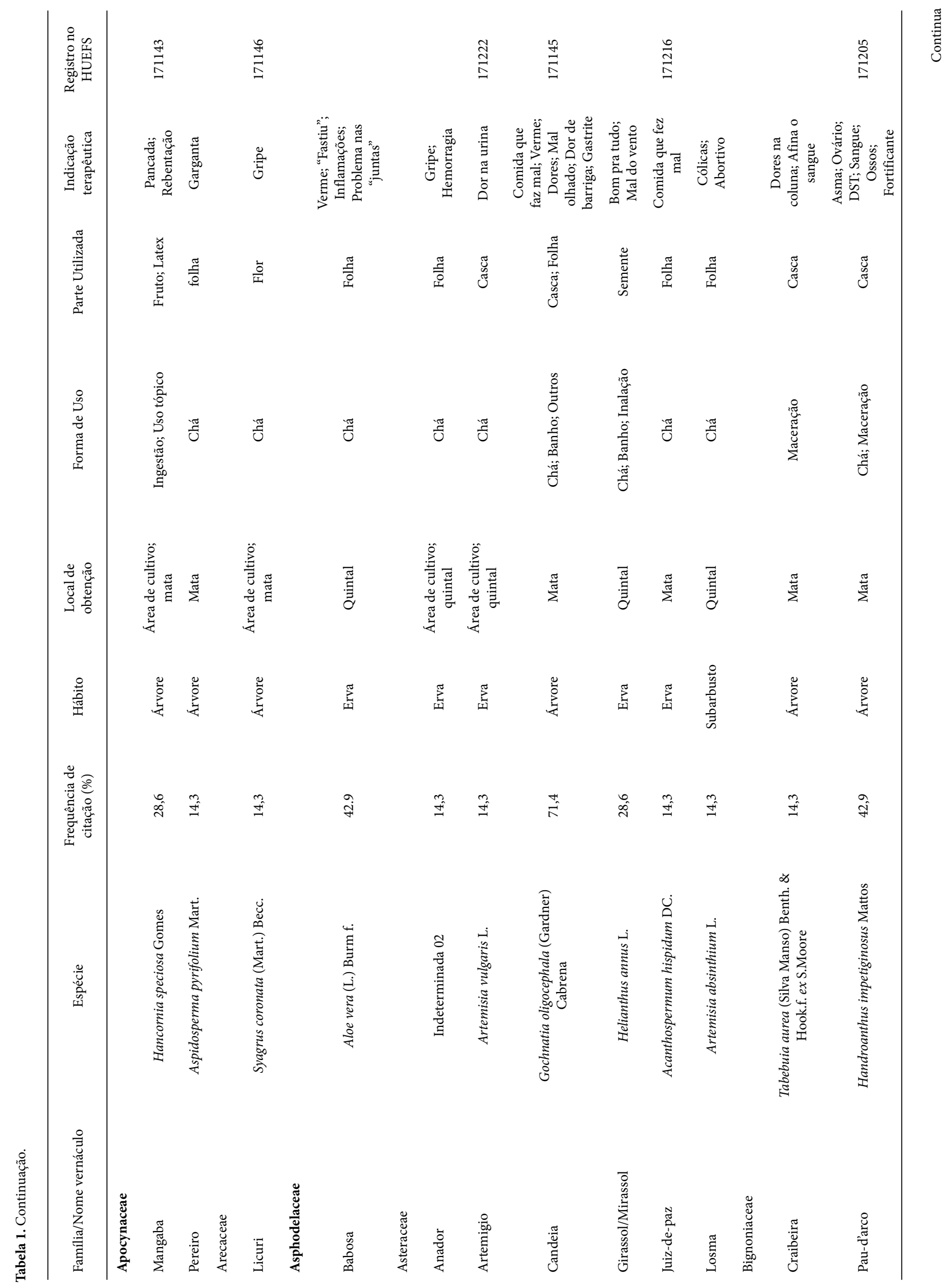




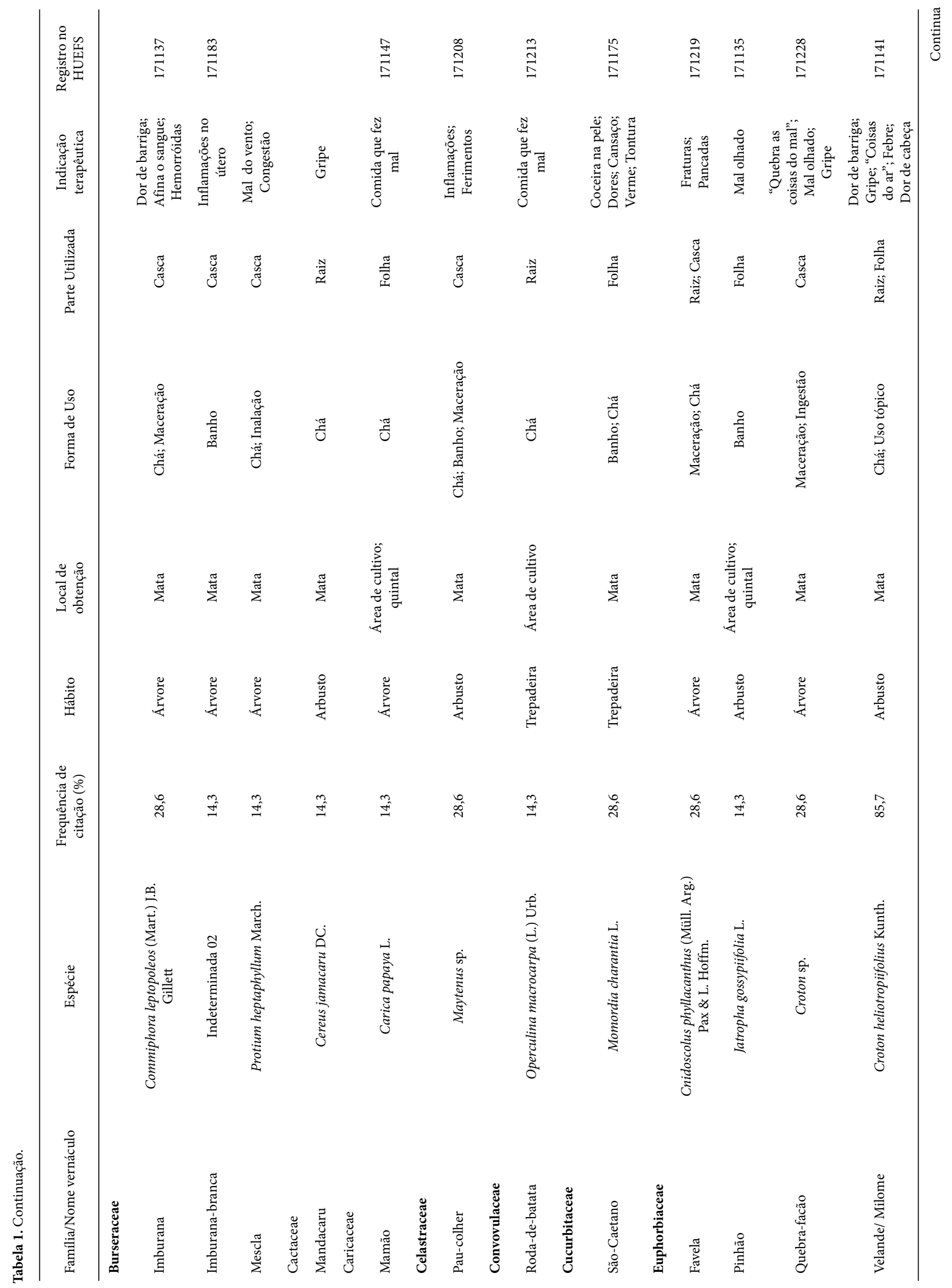




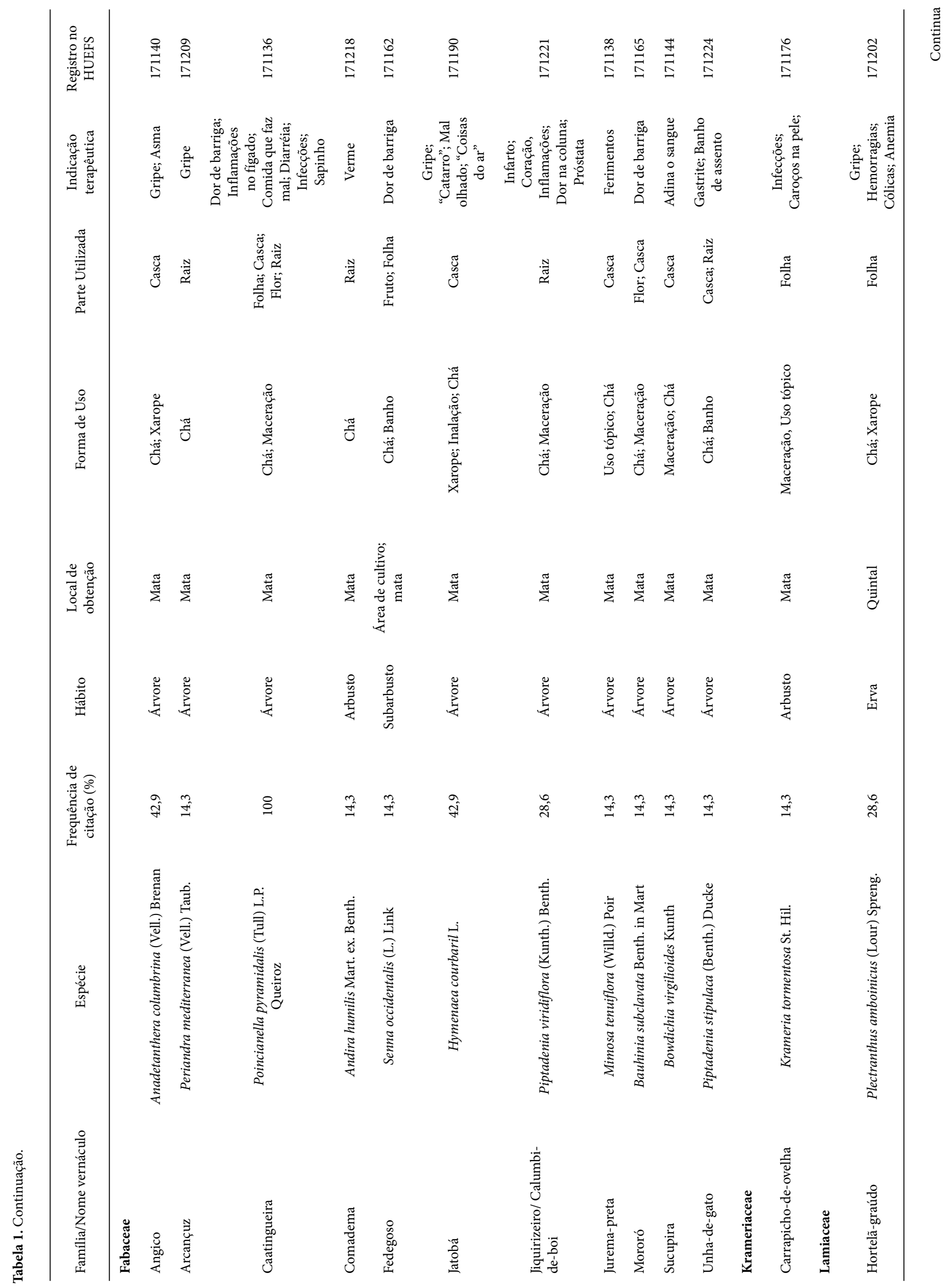




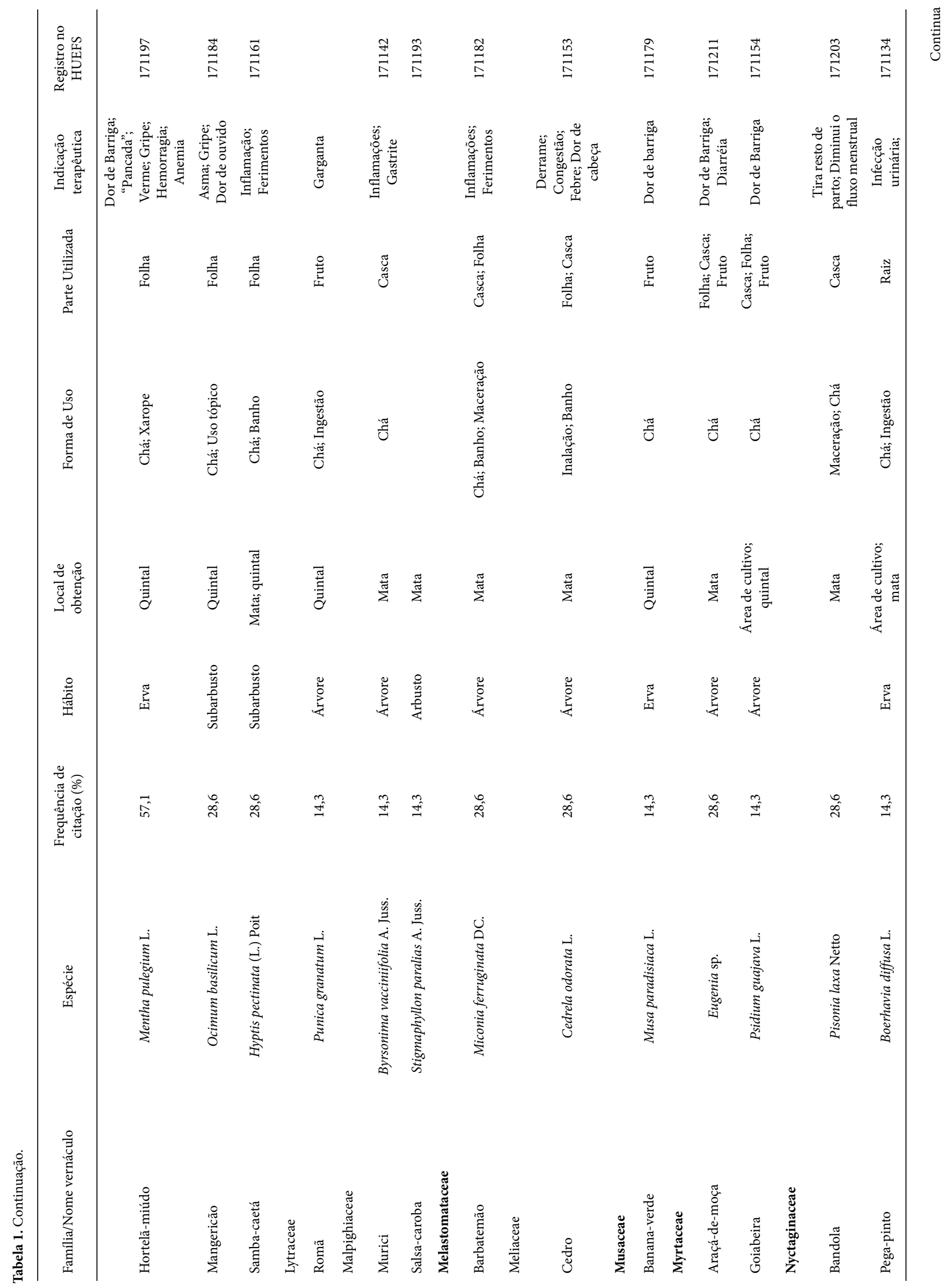




\begin{tabular}{|c|c|c|c|c|c|c|c|c|c|c|c|c|c|c|c|c|c|c|c|c|}
\hline 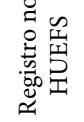 & $\stackrel{\vec{\infty}}{\underset{\Xi}{\Xi}}$ & & & 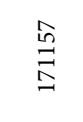 & & $\stackrel{i n}{\stackrel{1}{二}}$ & & & 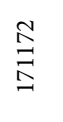 & & & & $\underset{\vec{n}}{\stackrel{\vec{n}}{\Xi}}$ & & $\begin{array}{l}\text { స్ } \\
\stackrel{\Xi}{\Xi}\end{array}$ & & & 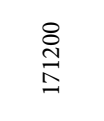 & & \\
\hline 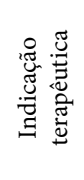 & 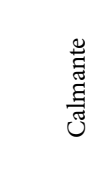 & 䒕 & & 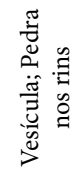 & & 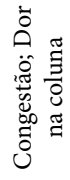 & & 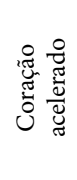 & 䞨 & 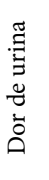 & 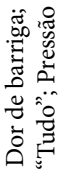 & & 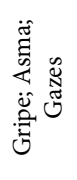 & & 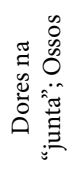 & 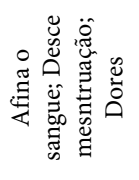 & & 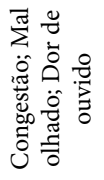 & 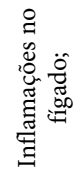 & \\
\hline 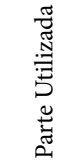 & 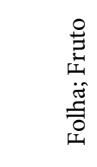 & 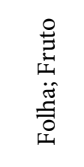 & & $\stackrel{\frac{g}{7}}{\circ}$ & & 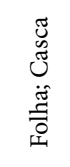 & & 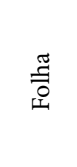 & 䍃 & $\stackrel{\vec{\partial}}{\Psi}$ & $\begin{array}{l}\stackrel{5}{0} \\
\text { क्ष }\end{array}$ & & $\begin{array}{l}\widetilde{J} \\
\tilde{J}\end{array}$ & & $\begin{array}{l}\widetilde{J} \\
\text { J } \\
\text { Uू }\end{array}$ & 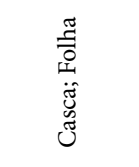 & & 㺃 & $\begin{array}{l}\mathbb{J} \\
\text { J } \\
\tilde{J}\end{array}$ & \\
\hline 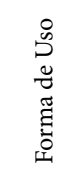 & 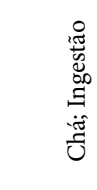 & 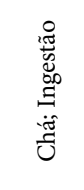 & & 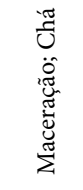 & & 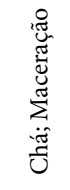 & & ]ु & 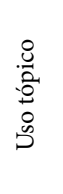 & 窇 & త্ & & త్ & & 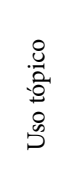 & 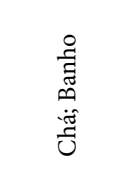 & & 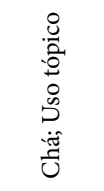 & త్ & \\
\hline 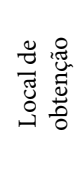 & $\frac{\pi}{2}$ & 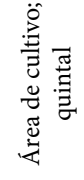 & & 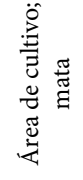 & & 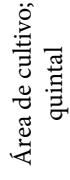 & & 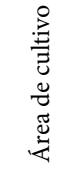 & $\stackrel{\frac{\tilde{J}}{\Sigma}}{\Sigma}$ & $\frac{\pi}{2 \pi}$ & 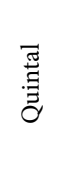 & & ${ }_{\frac{\pi}{\pi}}^{\frac{\pi}{\pi}}$ & & 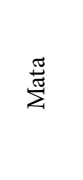 & $\sum_{\frac{\pi}{\pi}}^{\frac{\pi}{\pi}}$ & & $\begin{array}{l}\text { 吾 } \\
\text { 音 }\end{array}$ & $\stackrel{\frac{\pi}{\pi}}{\Sigma}$ & \\
\hline 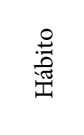 & 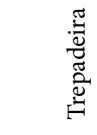 & 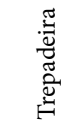 & & 䍃 & & 嶭 & & 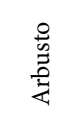 & 畄 & 嵒 & 䍃 & & 荌 & & 苛 & 蒙 & & 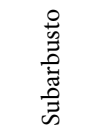 & 总 & \\
\hline 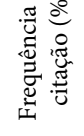 & $\stackrel{m}{ \pm}$ & $\stackrel{m}{\sharp}$ & & $\stackrel{m}{ \pm}$ & & $\stackrel{m}{ \pm}$ & & $\stackrel{m}{ \pm}$ & $\stackrel{m}{ \pm}$ & $\stackrel{m}{ \pm}$ & $\begin{array}{l}0 \\
\stackrel{0}{\sim} \\
\stackrel{0}{1}\end{array}$ & & $\stackrel{m}{ \pm}$ & & $\stackrel{m}{ \pm}$ & $\begin{array}{l}0 \\
\stackrel{0}{N}\end{array}$ & & $\begin{array}{l}\infty^{\circ} \\
\stackrel{\sim}{0}\end{array}$ & $\stackrel{m}{ \pm}$ & \\
\hline 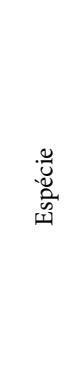 & 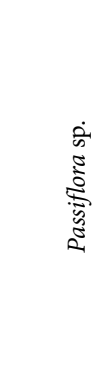 & 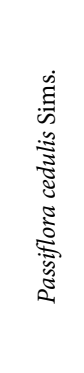 & & 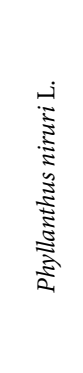 & & 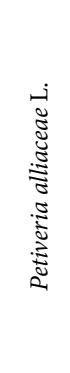 & & 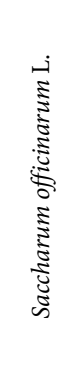 & 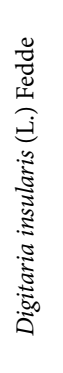 & 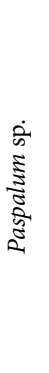 & 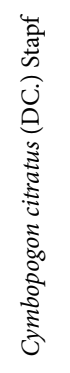 & & 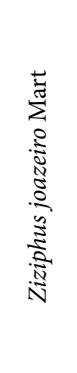 & & 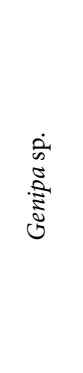 & 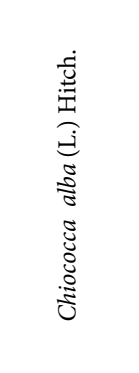 & & 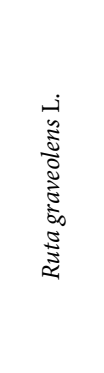 & 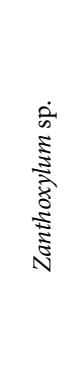 & \\
\hline 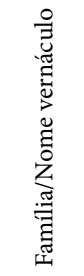 & 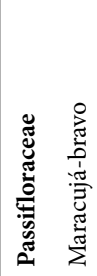 & 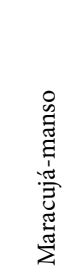 & 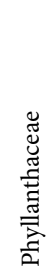 & 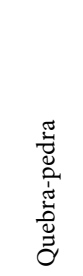 & 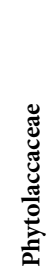 & 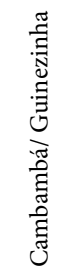 & 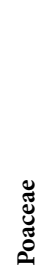 & 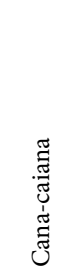 & 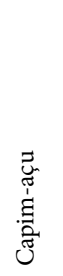 & 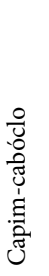 & 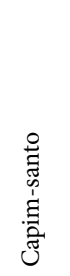 & 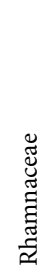 & 舀 & 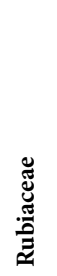 & 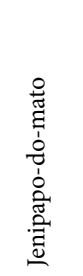 & 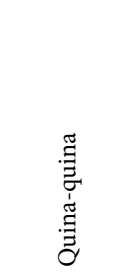 & 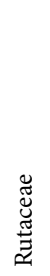 & 旁 & 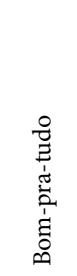 & 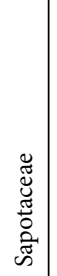 \\
\hline
\end{tabular}




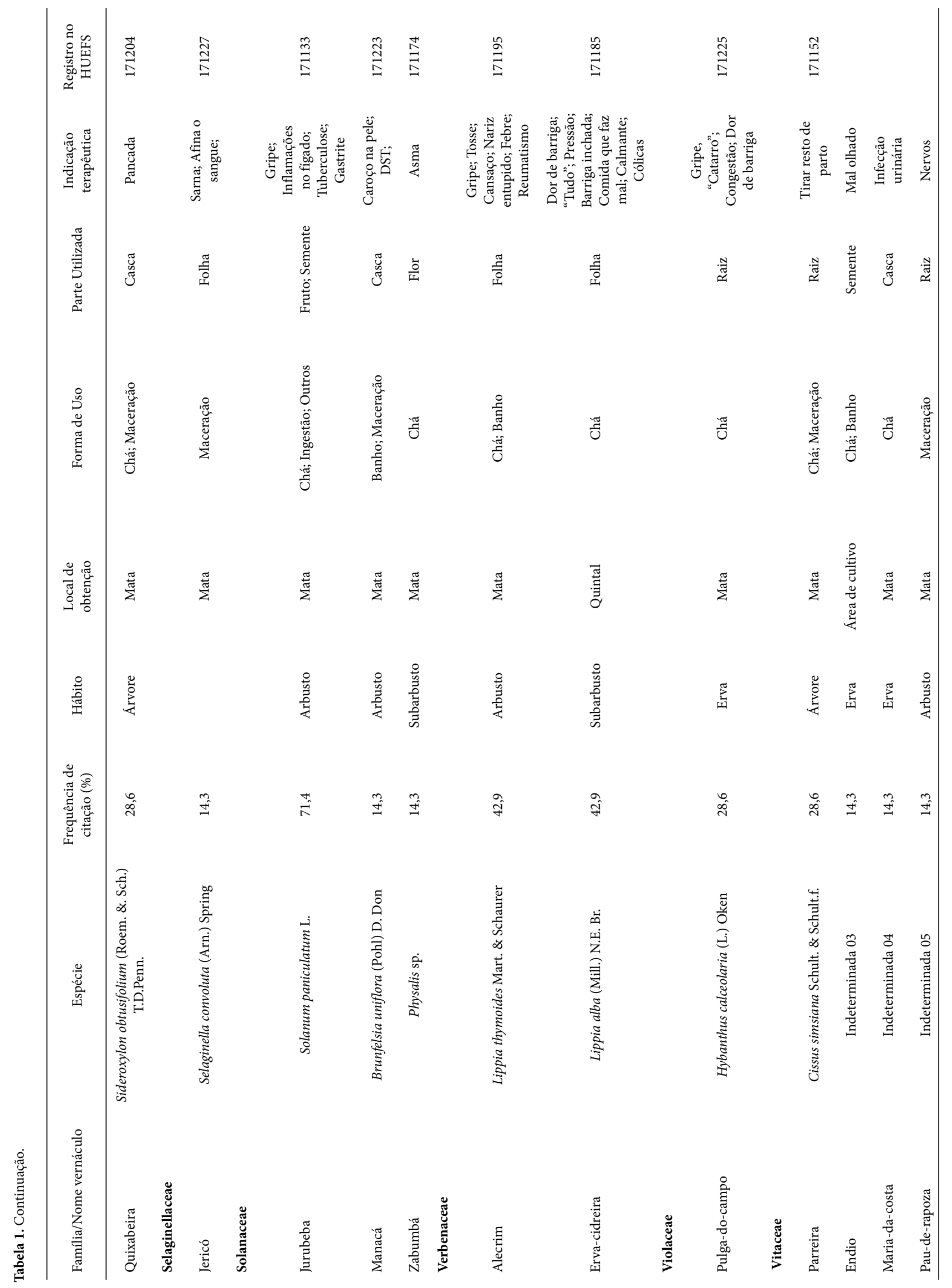


A espécie mais citada pelos colaboradores foi a catingueira (Poincianella pyramidalis), sendo indicada por $100 \%$ dos informantes, seguida pela aroeira (Schinus terebinthifolium) e o velande (Croton heliotropiifolius), indicadas por 86\% dos informantes; candeia (Gochnata oligocephala) e jurubeba (Solanum paniculatum) citadas por $71 \%$; hortelã miúdo (Mentha pulegium) citada por $57 \%$ dos informantes; alecrim (Lippia thymoides), angico (Anadenanthera columbrina), babosa (Aloe vera), braúna (Schinopsis brasiliensis), cajueiro (Anacardium accidentale), erva cidreira (Lippia alba), jatobá (Hymenaea coubaril), mastruz (Chenopodium ambrosioides) e pau d'arco (Handroanthus impetiginosus) foram citadas por $43 \%$ dos informantes. Analisando-se os dados observa-se que apenas a catingueira foi citada por todos informantes e que há poucas espécies (seis) citadas por mais de $50 \%$ dos informantes, por outro lado, a grande maioria das espécies é citada por dois ou apenas um informante. Isso significa que há, em relação ao conhecimento sobre o potencial medicinal das espécies utilizadas no povoado Casinhas, uma forte variação idiossincrática, em outras palavras, um grupo básico de plantas é lembrado ou reconhecido, não necessariamente mais utilizado, como medicinal pela maior parte dos entrevistados enquanto a maioria das plantas tem uma baixa freqüência de citação. Quanto ao local de obtenção das plantas, verificou-se que $55 \%$ das espécies citadas são encontradas na mata, 26\% são encontradas nos quintais e $19 \%$ em áreas de cultivo. Desse modo, uma pequena parte das espécies listadas pelos informantes é cultivada nos quintais ou nas áreas de plantio (roças) e a grande maioria é retirada das capoeiras ou tabuleiro (mata secundária próxima ao povoado), local onde se encontra grande diversidade de espécies arbóreas e nativas com grande importância para o uso e conservação das espécies nativas do bioma Caatinga (Albuquerque \& Andrade 2002).

A maioria das espécies foi indicada para doenças do sistema digestório, infecções e doenças do sistema respiratório. Resultados semelhantes foram encontrados em trabalhos realizados na região nordeste do Brasil (Monteles \& Pinheiro 2007), mais especificamente em áreas de Caatinga (Albuquerque et al. 2007; Albuquerque et al. 2006) bem como em outras regiões do Brasil (Amorozo 2002; Silva-Almeida \& Amorozo 1998; Hanazaki et al. 1996). Almeida \& Albuquerque (2002) afirmam que os dados obtidos em pesquisas dessa natureza estão diretamente relacionados à maioria dos casos de doenças na população. No estudo em questão, isso pode ser corroborado por comunicação pessoal da agente comunitária, membro do serviço de saúde local, ao informar que as doenças mais prevalentes na comunidade de Casinhas são: verminose, diarréia e falta de ar. Esse possível quadro epidemiológico reflete principalmente a ausência do Estado, refletido na de falta de políticas públicas voltadas para o saneamento básico, pois apesar da maioria das casas possuírem água encanada, não há nenhuma infra-estrutura para o tratamento da água que possibilite seu consumo seguro para a saúde bem como não há descarte adequado dos resíduos sólidos e efluentes domésticos. Tal quadro também pode estar relacionado, porém em menor intensidade, a questões culturais e educacionais relativas aos cuidados com a higiene pessoal nas zonas rurais.

Dentre as espécies citadas, o velande (Croton heliotropiifolius) foi a espécie de maior versatilidade, pois atende a cinco sistemas corporais: sistema digestório, infecções, sistema nervoso, sistema respiratório e doenças culturais. Em seguida, estão a aroeira (Schinus terebinthifolium), a braúna (Schinopsis brasiliensis), o cajueiro (Anacardium occidentale), o hortelã-

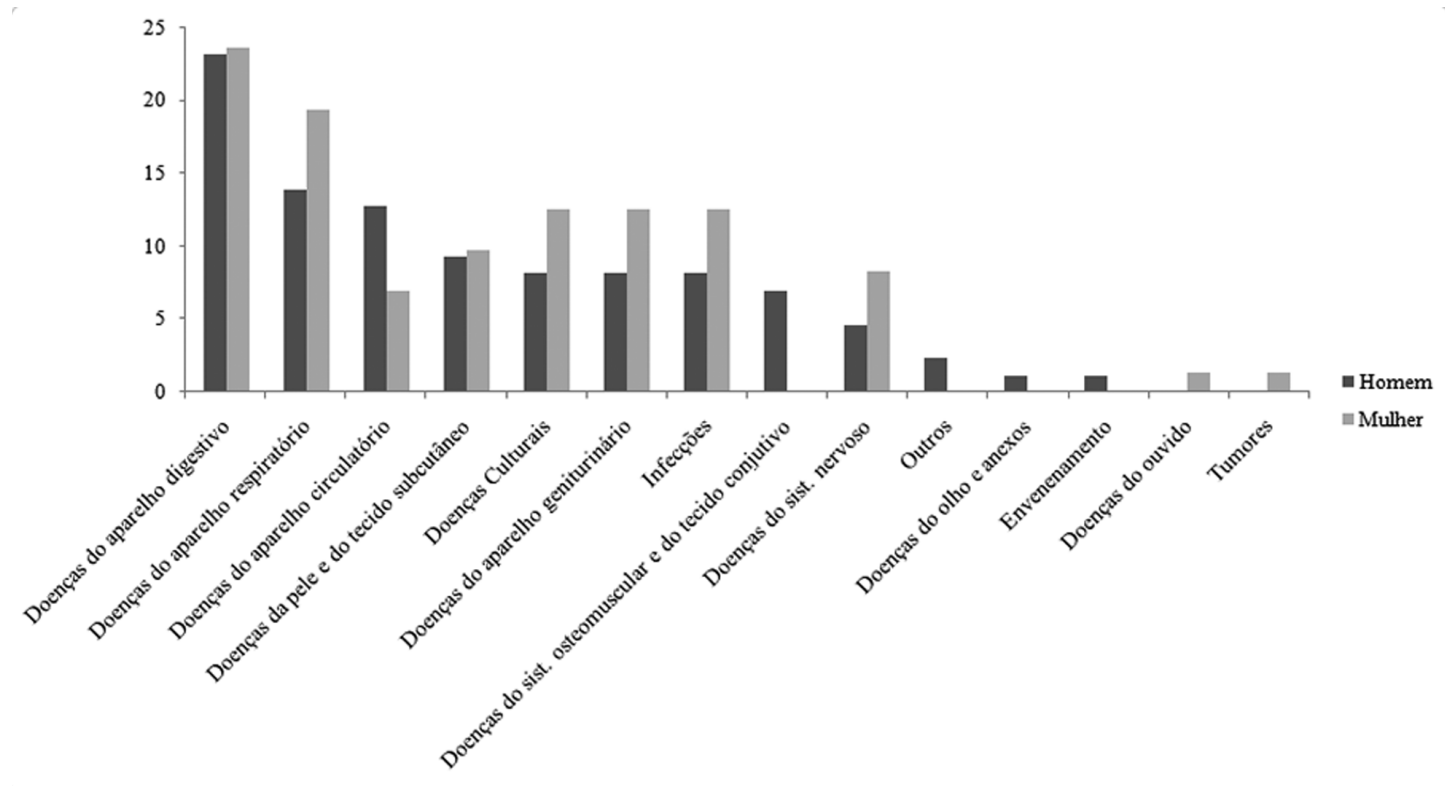

Figura 1. Número de espécies citadas por gênero para diferentes sistemas corporais, na comunidade Casinhas, Jeremoabo, BA. 
-miúdo (Mentha pulegium), o pau d'arco (Handroanthus impetiginosus) e o jiquirizeiro (Piptadenia viridiflora), com indicações para quatro sistemas. Dez espécies são indicadas para três sistemas, dezoito espécies para dois sistemas e as demais espécies apresentaram indicação para um único sistema corporal. É relevante destacar a indicação de espécies que são utilizadas em rituais de origem africana, pois apesar dos moradores da comunidade seguirem a religião católica ou protestante, tal prática ainda se mostra fortemente presente no cotidiano, como as rezas para a cura de doenças, "livramento do mal" e outras doenças "espirituais".

Quanto ao domínio do conhecimento e a questão de gênero, pode-se inferir que as mulheres tendem a demonstrar um maior conhecimento acerca da utilização das plantas medicinais do que os homens. Como pode ser visualizado na figura 1, as mulheres citaram um maior número de plantas medicinais para o tratamento de doenças que acometem mais de 50\% dos sistemas corporais referidos pelos entrevistados. Isso pode ser explicado porque as mesmas além dos cuidados com a casa, são lavradoras, mães e esposas, sendo responsáveis pelas estratégias de manutenção da saúde da família. Vale ainda ressaltar que, no que diz respeito às indicações terapêuticas na comunidade de Casinhas, existe uma ética, um respeito cultural às diferenças de conhecimento entre gêneros, de modo que os homens têm receio de indicar aquelas plantas que estão relacionadas às afecções femininas e vice-versa.

Ao analisar as indicações feitas nas entrevistas pelos homens e pelas mulheres de forma isolada, observa-se que algumas plantas como bandola (Pisonia laxa), losma (Artemisia absinthium), maria da costa (indeterminada 04), parreira (Cissus simsiana) e unha de gato (Piptadenia stipulaca) estão apenas relacionadas a problemas exclusivamente femininos, tais como: "descer menstruação", "tirar resto de parto", "banho de acento", cólicas, abortivos e foram indicadas apenas por mulheres. Franco \& Barros (2006) ao estudarem o quilombo Olho D'água dos Pires, no estado do Piauí, também obtiveram resultados similares. Já o artemigio (Artemisia vulgaris), a braúna (Schinopsis brasiliensis) e o calumbi de boi (Piptadenia viridiflora) foram citadas apenas por homens tendo sido indicadas para dores na próstata e dores ao urinar. Esse resultado revela também que existem diferenças de gênero na escolha das plantas, e esse padrão está relacionado às particularidades das atividades produtivas realizadas por homens e mulheres (Almeida \& Bandeira, 2010). Nas comunidades que estudaram Amorozo (1996) bem como Borba \& Macedo (2006) também observaram esse comportamento em relação ao domínio cognitivo entre os gêneros sobre o conhecimento de plantas. Na comunidade de Casinhas houve resistência para obter tais informações durante conversas informais, pois quando argüidos sobre $\mathrm{o}$ assunto, os entrevistados desconversam $\mathrm{e}$ pediam para procurar alguém do sexo oposto.

As folhas e as cascas são as partes mais citadas no preparo dos remédios caseiros, sendo referenciadas para $36 \%$ e $30 \%$ dos usos, respectivamente. Resultados semelhantes foram encontrados por Franco \& Barros (2006) no quilombo Olho Dágua dos Pires - PI; em Santo Antônio do Leveger - MT por Amorozo (2002) e no Cariri Paraibano por Agra et al. (2007). Em se tratando de Caatinga, alguns estudos relatam que a casca apresenta a maior porcentagem de citações (Albuquerque \& Andrade, 2002; Almeida \& Albuquerque 2002), isso é parcialmente explicado porque esta é uma das partes das plantas disponível ao longo de todo o ano. Gomes (2008) afirma que a utilização da casca é um dado preocupante, pois, se feita de forma insustentável, tal prática colocará em risco as populações das espécies, principalmente se forem nativas. Em Casinhas, no período de estiagem, a disponibilidade das folhas das plantas nativas é escassa, sendo priorizada a utilização da casca das árvores, enquanto no período chuvoso estão disponíveis tanto as espécies herbáceas quanto as folhas das espécies lenhosas. Assim há uma oferta de recursos vegetais diferenciada durante $o$ ano. Esta disponibilidade diferenciada pode ajudar a reduzir a pressão de coleta ao longo do ano das mesmas partes da planta. Além disso, na comunidade estudada a maioria das espécies, incluindo as ervas, recebem as mesmas indicações terapêuticas, desse modo pode haver uma alternância na escolha do recurso a ser utilizado, amenizando a pressão de coleta sobre um pequeno número de espécies. Foram citadas sete formas de preparo para as espécies utilizadas, sendo o chá é a forma mais utilizada pelos moradores da comunidade, correspondendo a $51 \%$ das citações, seguida pela maceração com $17 \%$ das citações. Resultados semelhantes foram encontrados nos estudos em um quilombo maranhense (Monteles \& Pinheiro 2007).

$\mathrm{O}$ valor de saliência das espécies utilizadas como medicinais no povoado Casinhas variou entre $0,09 \mathrm{e} 0,65$. As dez espécies que tiveram maior valor de saliência cultural, em ordem decrescente, foram: catingueira (Poincianella pyramidalis - 0,65), aroeira (Schinus terebinthifolius - 0,58), candeia (Gochinatia oligocephala - 0,45), jurubeba (Solanum paniculatum - 0,37), erva cidreira Lippia alba- 0,36), hortelã miúdo (Mentha pulegium - 0,35), cajueiro (Anacardium ocidentale - 0,32), milome (Croton heliotropiifolius - 0,29), mastruz (Chenopodium ambrosioides - 0,25) e jatobá (Hymenaea courbaril - 0,25). Almeida \& Bandeira (2010) em um estudo etnobotânico da comunidade de Casinhas, identificaram as espécies que tinham o maior valor local para a comunidade, considerando todos os tipos de usos. Das dez espécies que apresentaram maiores valores locais no estudo citado cinco apresentaram maior índice de saliência para este estudo. Observa-se ainda que aproximadamente 50\% das espécies inventariadas por Almeida \& Bandeira (2010) estão inseridas na presente pesquisa, mostrando que há uma plasticidade no uso dos recursos vegetais na área estudada bem como a importância dos mesmos para a comunidade.

Das 15 espécies que tiveram maior freqüência de citação dez delas apresentaram maior valor de saliência. Essa pequena diferença, mas não menos importante, se dá por conta 
do ordenamento e da seqüência em que as plantas foram citadas (ranking), assim as espécies que se mostraram mais salientes culturalmente estão entre as espécies que também são mais freqüentes.

Os dados apresentados mostram que a comunidade estudada conhece e depende estreitamente dos recursos vegetais para suas práticas médicas tradicionais, seja por questões culturais, econômicas ou pela dificuldade de acesso à medicina convencional. As espécies que apresentaram maior índice de saliência são, na grande maioria, espécies nativas, o que reforça a necessidade de maior atenção no desenvolvimento de programas de conservação da Caatinga. Os resultados aqui apresentados também podem ser utilizados como base para a bioprospecção; para estudos posteriores no desenvolvimento de fitomedicamentos, com a devida repartição de benefícios e consentimento prévio das comunidades, bem como instrumento para seleção de espécies prioritárias para estudos de ecologia de populações, visando o uso e manejo sustentável das mesmas. Os resultados dessa pesquisa também indicam que é de extrema importância que os conhecimentos tradicionais e práticas de cura dessas populações históricas, de quilombolas, sejam registrados e valorizados, mas, sobretudo que sejam considerados nas políticas públicas de saúde dirigidas a essas populações.

\section{Agradecimentos}

Ao curso de Ciências Biológicas da Universidade do Estado da Bahia - Campus VIII, ao qual esse projeto está vinculado, ao Herbário e aos botânicos especialistas da UEFS, em particular ao Dr. Luciano Paganucci de Queiróz. À Dra. Vanusa Almeida pelas sugestões e correções ao texto. Ao Dr. Jomar Jardim, curador do Herbário da Universidade Federal do Rio Grande do Norte, pela revisão dos nomes válidos das espécies bem como identificação de algumas. Às pessoas que direta ou indiretamente contribuíram para esta pesquisa. À comunidade Casinhas pelo acolhimento, receptividade, colaboração e contribuição que foram fundamentais para a realização deste trabalho.

\section{Referências bibliográficas}

Agra, M.F.; Baracho, G.S.; Basílio, I.J.D.; Nurit, K; Coelho, V.P. \& Barbosa, D.A. 2007 Sinopse da flora medicinal do Cariri paraibano. Oecologia Brasiliensis 11(3): 323-330.

Albuquerque, U.P. \& Andrade, L.H.C. 2002. Conhecimento botânico tradicional e conservação em uma área de Caatinga no estado de Pernambuco, Nordeste do Brasil. Acta Botanica Brasilica 16(3): 273-285.

Albuquerque, U.P.; Araújo, T.A.S.; Ramos, M.A.; Nascimento, V.T.; Lucena, R.F.P.; Monteiro, J,M.; Alencar, N.L. \& Araújo, E.L. 2009. How ethnobotany can aid biodiversity conservation: reflections on investigations in the semiarid region of NE Brazil. Biodiversity and Conservation 18: 127-150.

Albuquerque, U.P.; Medeiros, P.M.; Almeida, A.L.S.; Monteiro, J.M.; Neto, E.M.F.L.; Melo, J.G. \& Santos, J.P. 2007. Medicinal plants of the Caatinga (semi-arid) vegetation of NE Brazil: A quantitative approach. Journal of Ethnopharmacology 114: 325-354.

Albuquerque, U.P. 2005. Introdução à Etnobotânica. 2 ed. Rio de Janeiro, Interciência.
Albuquerque, U.P. 2001. The use of medicinal plants by cultural descendants of African people in Brazil. Acta Farmaceutica Bonaerense 20(20): 139-144

Alexiades, M. 1996. Selected guidelines for ethnobotanical research: a field manual. New York, New York Botanical Garden.

Almeira, C.F.C.B.R.; Amorim, E.L.C.; Albuquerque, U.P.; \& Maia, M.B.S. 2006. Medicinal plants popularly used in Xingó region - a semi-arid location in Northeastern Brazil. Journal of Ethnobiology and Ethnomedicine 2(15): 1-9.

Almeida, C.F.C.B.R. \& Albuquerque, U.P. 2002. Uso e conservação de plantas e animais medicinais no estado de Pernambuco (Nordeste do Brasil): Um estudo de caso. Interciencia 27(6): 276-285.

Almeida, T.F. \& Rodrigues, A.C.C. 2006. Etnobotânica das plantas medicinais de Madre de Deus, Bahia. Sitientibus Série Ciências Biológicas 6(2): 133-137.

Almeida, V.S. \& Bandeira, F.P.S.F. 2010. O significado cultural do uso de plantas da caatinga pelos quilombolas do Raso da Catarina, município de Jeremoabo, Bahia, Brasil. Rodriguesia 61(2): 195-209.

Amorozo M.C.M. 1996. A abordagem etnobotânica na pesquisa de plantas medicinais. Pp. 47-68. In: Di Stasi, L.S. (Ed.). Plantas medicinais: arte e ciência. Um guia de estudo interdisciplinar. São Paulo, Ed. UNESP.

Amorozo, M.C.M. 2002. Uso e diversidade de plantas medicinais em Santo Antônio do Leveger, MT, Brasil. Acta Botanica Brasilica 16(2): 189-203.

Anjos, R.S.A. 2000. Territórios das Comunidades Remanescentes de Antigos Quilombos no Brasil - Primeira Configuração Espacial. 2. ed. Brasília, Mapas Editora \& Consultorias.

APG III. 2009. An update of the Angiosperm Phylogeny Group classification for the orders and families of flowering plants: APG III. Botanical Journal of the Linnean Society 161: 105-121.

Bailey, K. 1994. Methods of social research. 4 ed. New York, The Free Press.

Bernard, H.R. 1998. Redearch methods in cultural antropology. 2 ed USA, SAGE Publications.

Borba, A.M. \& Macedo, M. 2006. Plantas medicinais usadas para saúde bucal pela comunidade do bairro Santa Cruz, Chapada dos Guimarães, Mato Grosso, Brasil. Acta Botanica Brasilica 20: 771-782.

Borgatti, S.P. 1992. ANTHROPAC 4.00 methods guide. Columbia, Analytic Technologies.

CEI. 1994. Centro de estatística e informacões-básicas dos municípios baianos: região nordeste 11: 35-37.

Franco, E.A.P. \& Barros, R.F.M. 2006. Uso e diversidade de plantas medicinais no Quilombo Olho D'água dos Pires, Esperntina, Piauí. Revista Brasileira de Plantas Medicinais 8(3): 78-88.

Gomes, E.C.S.; Barbosa, J.; Vilar, F.C.R.; Peres, J.O.; Vilar, R.C.; Freire, J.L.O.; Lima, A.N.L. \& Dias, T.J. 2008. Plantas da caatinga de uso terapêutico: Levantamento etnobotânico. Engenharia Ambiental 5(2): 74-85

Gottlieb, S.L.; Borin, M.R.M.B.; Pagotto, C.L.A.C.\& Zocher, D.H.T. 1998. Biodiversidade: o enfoque interdisciplinar brasileiro. Ciência e Saúde Coletiva 3(2): 97-102.

Guimarães, I.M. 2009. O uso social das plantas: a prática da medicina popular no povoado Mussuca, comunidade quilombola em Laranjeiras (SE). In: Encontro de Ciências Sociais do Norte Nordeste. Recife.

Hanazaki, N.; Leitão-Filho, H. F. \& Begossi, A. 1996. Uso de recursos na mata atlântica: o caso do Pontal do Almada (Ubatuba, Brasil). Interciencia 21(6): 268-276.

IBGE-Instituto Brasileiro de Geografia e Estatística. 2008 (29 de agosto de 2008). < http://www.ibge.gov.br/home/estatistica/populacao/estimativa2008/POP2008_DOU.pdf> (Acesso em 05/09/2008).

IBAMA - Instituto Brasileiro do Meio Ambiente e dos Recursos Naturais Renováveis. 2005. Estação Ecológica Raso da Catarina. Disponível em: <//http://www.ibama.gov.br/siucweb/mostraUC/>. (Acesso em $17 / 11 / 2005)$.

MMA - Ministério do Meio Ambiente, dos Recursos hídricos e da Amazônia Legal. 2002. Avaliação e ações prioritárias para conservação da biodiversidade da Caatinga. Brasília, Universidade Federal de Pernambuco, Conservation International do Brasil e Fundação Biodiversitas.

Monteles, R. \& Pinheiro, C.U.B. 2007. Plantas medicinais em um quilombo maranhense: uma perspectiva etnobotânica. Revista de Biologia e Ciências da Terra 7(2): 38-48.

Mori, S.A.; Silva, L.A.M.; Lisboa, G. \& Coradin, L. 1989. Manual de manejo do herbário fanerogâmico. Ilhéus, Centro de Pesquisa do Cacau.

OMS - Organização Mundial da Saúde. 2000. Classificação Estatística Internacional de Doenças e Problemas Relacionados à Saúde 10(1): 1191. 
PDRS - Programa de Desenvolvimento Regional Sustentável Companhia de Desenvolvimento e Ação Regional. 2000. Salvador, CAR (BA) Nordeste da Bahia.

Quinlan, M. 2005. Considerations for collecting freelists in the Field: Examples from ethnobotany. Field Methods 17(3): 1-16.

Schmitt, A.; Turatti, M.C.M. \& Carvalho, M.C.P. 2002. A atualização do conceito de quilombo: identidade e território nas definições teóricas. Ambiente e Sociedade 10: 129-136.

Schultes, R.E. \& Reis, S.V. 1995. Ethnobotany: evolution of a discipline. Pp. 19-39. 1. ed. Cambridge, Dioscorides Press.

Silva-Almeida, M.F. \& Amorozo, M.C.M. 1998. Medicina popular no Distrito de Ferraz, Município de Rio Claro, Estado de São Paulo. Brazilian Journal of Ecology 2: 36-46.
Simões, C.M.O.; Mentz, L.A.; Schenkel, E.P.; Irgang, B.E. \& Stehmann, J.R. 1998. Plantas medicinais populares no Rio Grande do Sul. 3. ed. Porto Alegre, Ed. Univers.

Teixeira, S.A. \& Melo, J.I.M.. 2006. Plantas medicinais utilizadas no município de Jupi, Pernambuco, Brasil. Iheringia, Série Botânica 61(1-2): 5-11.

Toledo, V.M. Biodiversity and indigenous peoples. Pp. 330-340. In: Levin, S.A. (Ed.). Encyclopedia of Biodiversity. San Diego, Academic Press.

Vieira, F.J.; Santos, L.G.P; Araújo, J.L.L. \& Barros, R.F.M. 2008. Quilombola of Macacos community, São Miguel do Tapuio City, Piauí State: History, use and conservation of plant resourses. Funtional Ecosystems and Communities 2: 81-87.

Weller, S.C. \& Romney, K.A. 1988. Systematic data collection. Newbury Park, CA: Sage. 